\title{
Uncovering Structure-Properties Relations in Fuel Cells and Catalysts with Quantitative Aberration-Corrected STEM and EELS
}

\author{
Jae Hyuck Jang, ${ }^{1}$ Qian He, ${ }^{1}$ Young-Min Kim, ${ }^{2}$ Donovan N. Leonard, ${ }^{1}$ Amit Kumar, ${ }^{3}$ Sergei V. \\ Kalinin, ${ }^{3}$ Albina Y. Borisevich ${ }^{1}$ \\ ${ }^{1}$ Materials Science and Technology Division, Oak Ridge National Laboratory, Oak Ridge, TN 37831, \\ USA \\ ${ }^{2}$ Division of Electron Microscopic Research, Korea Basic Science Institute, Daejeon 305-333, Korea \\ ${ }^{3}$ Center for Nanophase Materials Sciences, Oak Ridge National Laboratory, Oak Ridge, TN 37831 , \\ USA
}

Advances in materials science of fuel cells and catalysts promise global benefits: increased efficiency in power generation, decreased harmful emissions, and reduced energy use. Keys to fundamental understanding of the behavior of these materials, however, often lie on nano- or even atomic scales: functionality of solid oxide fuel cells is ultimately controlled by the static and dynamic behavior of oxygen vacancies; catalyst properties are governed by configuration and chemical composition of active centers. It is therefore crucial to be able to study relevant aspects of material behavior with atomic resolution. Recent advances in aberration-corrected electron microscopy and spectroscopy provide a wealth of structural and chemical information: local structure can be characterized with picometer precision [1], chemical identity of a single atom can be determined [2], and concentration of oxygen vacancies can be measured for every unit cell [3]. Scanning transmission electron microscopy (STEM) setting is especially useful since multiple types of signals can be collected simultaneously and correlated. These methods can be applied in both in situ and ex situ configurations.

This approach has proven very successful for studies of nitrogen doped carbon/ Pt electrocatalysts, revealing the mechanism of properties improvement due to nitrogen addition and enabling optimization of the synthesis procedures (Fig. 1(a)) [4]. In Mo-V-Te-(Nb,Ta)-O catalysts, cation distribution could be quantitatively determined (Fig. 1(b))[5]. Studies of the surfaces of these catalysts uncovered surface decoration by different structural phases, which in turn helped explain synergetic effects in catalyst selectivity [6].

In fuel cells, oxygen vacancy mapping offers multiple opportunities. Interestingly, many recent results illustrate that reversible vacancy redistribution in oxides is possible at room temperature and below in sufficiently high electric fields [7], suggesting that we can study these phenomena inside electron microscope, using bias or electron beam as excitation. For example, we can trace the improved performance of the lanthanum strontium cobaltite-based fuel cell anodes to surface amorphization and observe "annealing" of a vacancy ordered system caused by bias cycling [8]. We can also observe vacancy ordering and injection under the influence of electron beam; using quantitative imaging, we can determine the degrees of ordering and injection as a function of time and map out reaction pathways. We can study different types of samples and delineate the factors governing vacancy injection speed. We can recreate reaction sequences known from the bulk and collect information about local mechanisms of phase transitions [9]. Finally, prospects of further development of the technique and expected benefits will be discussed [10]. 


\section{References:}

[1] C.L. Jia et al., Nature Mater, 6, 64 (2007)

[2] O.L. Krivanek et al., Nature 464, 571 (2010)

[3] Y.M. Kim et al., Nature Mater., 11, 888 (2012).

[4] S. Pylypenko et al., Energy Environ. Science, 6, 2957 (2013).

[5] J. Yu et al., Catalysis Communications 29, 68-72 (2013).

[6] Q. He et al., these proceedings

[7] J. Fleig et al., Scripta Materialia 65, 78 (2011)

[8] D.N. Leonard et al., Adv. Energy Mater., 3, 788 (2013)

[9] J.H. Jang et al., these proceedings

[10] Research supported by the Materials Science and Engineering Division, U.S. Department of Energy (DOE), and through user projects supported by ORNL's Center for Nanophase Materials Sciences, sponsored by the Scientific User Facilities Division, Office of Basic Energy Sciences, U.S. DOE.
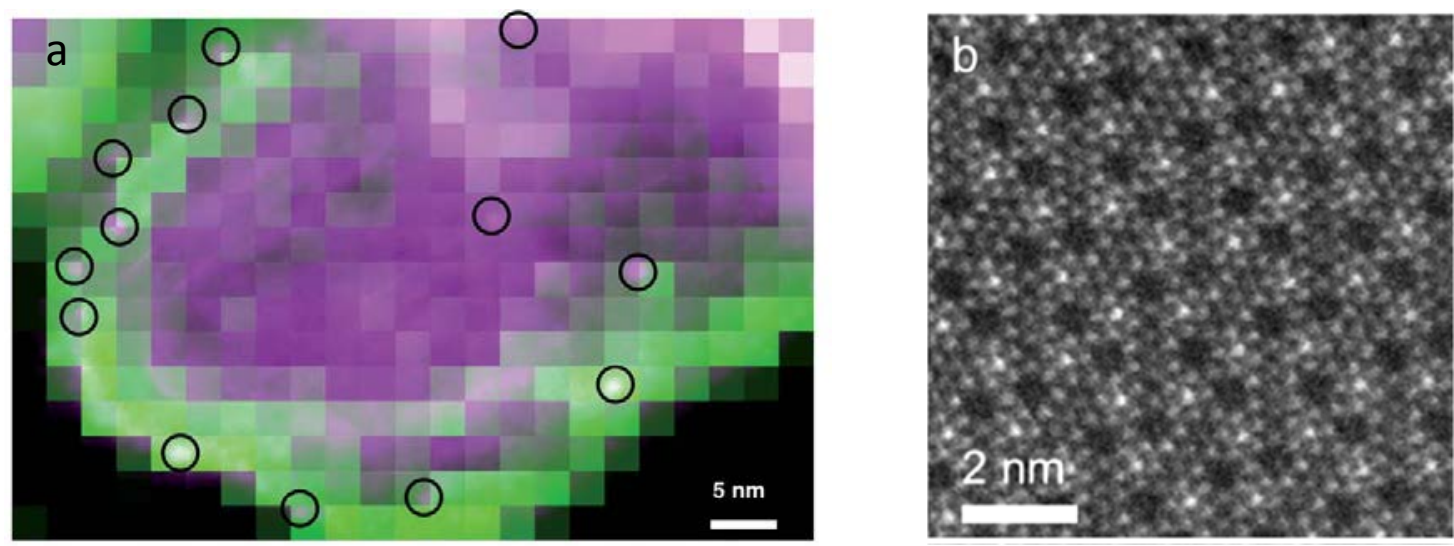

Figure 1 (a) Overlapped HAADF signal (purple) and N K EEL edge integrated signal (green) demonstrating attachment of Pt particles (circled in black) close to places of nitrogen absorption on activated carbon substrate (from [4]). (b) HAADF STEM image of Mo-V-Te-Ta-O M1 phase; bright spots are Ta atoms (from [5]).
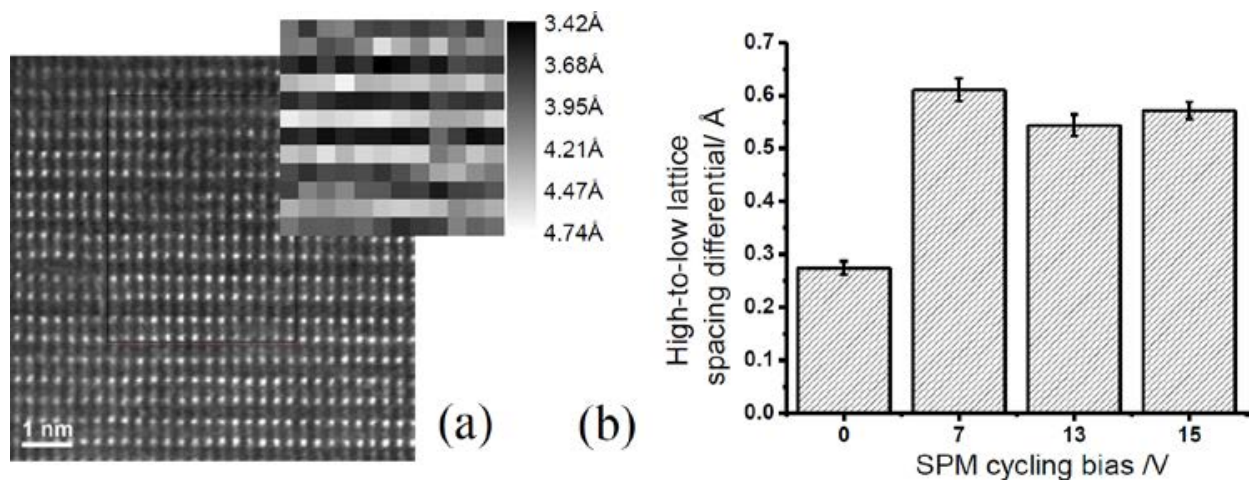

Figure 2 Vacancy ordering in LSCO and its changes after bias cycling. (a) A typical STEM image of a vacancy ordered region of LSCO; note modulated image contrast and lattice spacings. Inset illustrates lattice spacing map computed from the image. (b) The difference between the highest and the lowest spacings in a map such as inset in Figure 9(a) is a measure of the degree of order. This difference is plotted for areas cycled at different bias values. Degree of order clearly increases for cycled samples. The $0 \mathrm{~V}$ data is acquired from a different (uncycled) LSCO electrode (from [8]). 